\title{
Second-Order Conic Programming Model for Load Restoration Considering Uncertainty of Load Increment based on Information Gap Decision Theory
}

Xie, Yunyun; Xi, Chen; Wu, Qiuwei; Qian, Zhou

Published in:

International Journal of Electrical Power \& Energy Systems

Link to article, DOI:

10.1016/j.ijepes.2018.08.018

Publication date:

2019

Document Version

Peer reviewed version

Link back to DTU Orbit

Citation $(A P A)$ :

Xie, Y., Xi, C., Wu, Q., \& Qian, Z. (2019). Second-Order Conic Programming Model for Load Restoration Considering Uncertainty of Load Increment based on Information Gap Decision Theory. International Journal of Electrical Power \& Energy Systems, 105, 151-158. https://doi.org/10.1016/j.ijepes.2018.08.018

\section{General rights}

Copyright and moral rights for the publications made accessible in the public portal are retained by the authors and/or other copyright owners and it is a condition of accessing publications that users recognise and abide by the legal requirements associated with these rights.

- Users may download and print one copy of any publication from the public portal for the purpose of private study or research.

- You may not further distribute the material or use it for any profit-making activity or commercial gain

- You may freely distribute the URL identifying the publication in the public portal 


\title{
SciVerse ScienceDirect
}

00 (2012) 000-000

\section{Second-Order Conic Programming Model for Load Restoration Considering Uncertainty of Load Increment based on Information Gap Decision Theory}

\author{
Xie Yunyun ${ }^{\mathrm{a}}$, Chen Xi ${ }^{\mathrm{a}}$, Wu Qiuwei ${ }^{\mathrm{b}, \mathrm{d}} *$, Zhou Qian ${ }^{\mathrm{c}}$ \\ ${ }^{a}$ School of Auotmation, Nanjing University of Science and Technology, Nanjing 210094, Jiangsu Province, China \\ ${ }^{b}$ Centre for Electric Power and Energy, Technical University of Denmark, Kgs. Lyngby, DK 2800 \\ ${ }^{c}$ Electric Power Research Institute of State Grid Jiangsu Electric Power Company, Nanjing 211003, Jiangsu Province, China \\ $d$ Harvard China Project, School of Engineering and Applied Sciences (SEAS), Harvard University,29 Oxford Street, Cambridge, 02138 \\ MA, USA
}

\begin{abstract}
Load restoration is an important issue for power system restoration after a blackout. A second order conic programming (SOCP) model is proposed based on the information gap decision theory (IGDT) to maximize load pickup considering the uncertainty of load increment. Because distribution functions of load increment are difficult to obtain, the optimization of load pickup is transformed to maximize the fluctuation range of load increment by the IGDT. The derived optimal fluctuation range can ensure that the reenergized system is secure, and the amount of load pickup is always better than the specified expectation. Moreover, because the optimization model of the fluctuation range is a mixed-integer nonlinear model which is challenging to solve accurately and efficiently, the nonlinear model is transformed into a SOCP model that can be efficiently solved using CPLEX. The efficiency of the IGDT-based SOCP model is validated using the New England (10-machine 39-bus) system. The simulation results show that the derived load pickup shows expected robustness with respect to the load increment uncertainty.
\end{abstract}

Key Word: Information gap decision theory, load restoration, mixed-integer second-order conic program, uncertainty.

\section{Introduction}

Load restoration is an important issue for power system restoration after a blackout. Large-area blackouts of a power system have occurred many times in the past decade, e.g., the Indian blackout in 2012 [1] and the Japanese blackout in 2010 [2], because power systems are operating close to their limits as a result of the deregulation of the power system [3]. In order to restore the systems quickly and safely, the restoration

\footnotetext{
* Corresponding author: Qiuwei Wu.
}

E-mail address: qw@elektro.dtu.dk. 
procedure of a power system is commonly divided into three stages: preparation, system restoration and load restoration [4]. In the system restoration stage and load restoration stage, load pickup can balance the output of generators and provide frequency and a voltage profile for the restored system. Load pickup can be optimized to maximize restored loads with the operational constraints of the power system and generators to reduce the blackout duration time of loads [5]-[6].

A lot of research has been conducted to optimize the load pickup. Because the load is picked up discretely, load restoration may result in frequency deviations, transient voltage sags, and power flows over the limit. The load behaviour characteristics of various types of industry loads were modeled in [7], [8]. An assessment method of the frequency response of cold load pickup in the restoration was proposed in [9]. The optimization model of load pickup for a specific substation was established in [5], considering the security constraints of dynamic frequency and voltage, steady-state power flow and cold load pickup. A battery storage system was employed in [10] to maximize the load pickup by maintaining frequency stability. The load restoration was optimized using a multi-agent consensus system in [11]. The penalty costs were considered in [12] to decrease the load restoration time. A two-stage hierarchical optimal model of load restoration was built in [13] based on synchrophasor technology. The induction of the decision tree was employed to select the load pickup sequence in [14].

In the above research, the actual load pickup in each step of the load restoration is assumed to be the same as the forecasted load pickup. However, when the distribution feeders with loads are restored, some devices may not restart, .g., some motors will not work immediately after the restoration of power supply; some devices may consume more power than normal operation, e.g., the air conditioner may consume more power than normal operation. Consequently, the actual load increment fluctuates around the forecasted load increment. A large difference between the actual and forecasted load increment may result in a security problem in the restored systems and decrease the efficiency of system restoration. For example, during the system restoration following the "8-14" blackout in the USA and Canada in 2003, an emergency demand response plan was implemented by the New York Independent System Operator (ISO), and 300 MW of load was shed because the actual load pickup was larger than the forecasted load pickup [15]. Benefitting from the successful implementation of the emergency control, the system restoration was only delayed by the accident. If the emergency control is not implemented properly, then the restored system may fail again. Consequently, it is necessary to optimize the load pickup considering the uncertainty of the load pickup.

The challenge of considering the uncertainty of load pickup is the modeling of uncertainty. Because of the challenges of modeling, the wide area monitoring system (WAMS), which can monitor the system parameters and provide more accurate measurements of the restored load, was employed to reduce the time step of load restoration and decrease the uncertainty of load pickup in [6]; however, the forecasted load increment was different from the actual load increment. The fuzzy chance constraint model was used to describe the uncertainty of load pickup in [16]; however, the accurate fuzzy parameters were challenging to obtain in the application of this method. Apart from fuzzy arithmetic, various methods exist for handling uncertainty, such as the probabilistic method and robust method, which are frequently used in power system dispatching [17]. Due to the lack of historical data, the probability density function is challenging to obtain, whereas the Information gap decision theory (IGDT) requires less prior knowledge of the uncertainties, making it more suitable for optimizing load pickup, which has been applied to power system operation with uncertain parameters [18]-[19]. In [20], the IGDT was employed to restore distribution networks considering the uncertainty of load. However, because the system characteristics, restoration requirements, restoration model for load restoration during transmission system restoration are different from distribution network restoration, the method in [20] cannot be directly applied to the load restoration in this paper.

The contribution of this paper is proposing an IGDT-based second-order conic programming (SOCP) model to optimize the load restoration considering uncertainty of load increment. Specifically, according to 
the IGDT, the optimization of load pickup is transformed to maximize the fluctuation range of the load increment in which the reenergized system can remain secure and the amount of load pickup is always better than a specified expectation. Furthermore, the nonlinear model of fluctuation range optimization is transformed into an SOCP model that can be efficiently solved using CPLEX. Compared to the fuzzy method and probabilistic method, the IGDT-based SOCP model requires little information of the probability distribution function of the load increment fluctuation and can efficiently determine the load pickup scheme.

The rest of the paper is organized as follows. Section II describes the robust optimization model of load restoration by IGDT. Section III presents details on the construction and solution of the SOCP model. The simulation results are presented in Section IV, followed by the conclusion.

\section{Problem Formulation}

To address the uncertainty of load increment for load restoration, an IGDT-based load restoration model is established to optimize the load pickup in this section.

\subsection{Overview of IGDT model}

A typical optimization model can be described as follows:

$$
\left\{\begin{aligned}
\max _{d} B(X, d) \\
\text { s.t. } H(X, d)=0 \\
G(X, d) \leq 0
\end{aligned}\right.
$$

where $X$ refers to the vector of input parameters representing the load increment in different load nodes in this study, $d$ refers to the vector of decision variables representing the restoration states of different load nodes, $B(X, d)$ refers to the optimization objective that maximize the load pickup, and $H(X, d)=0$ and $G(X, d) \leq 0$ are the equality and inequality constraints, respectively.

When the input parameter $X$ is an uncertain load increment, this parameter can be modeled in several forms using the IGDT [21]. The envelope bound model is employed in this study. Assuming that $\tilde{X}$ is the forecasted amount of uncertain load increment $X$ and $\alpha$ is the uncertain range of $X$, the model of $X$ can be expressed as,

$$
\left\{\begin{array}{l}
X \in U(\alpha, \tilde{X}) \\
U(\alpha, \tilde{X})=\{X:|(X-\tilde{X}) / \tilde{X}| \leq \alpha\}
\end{array}\right.
$$

where $U(\alpha, \quad X g)$ is the set of all values of $X$ whose deviation range from $X /$ is less than $\alpha$.

When the value of uncertain load increment is equal to the forecasted value, the objective value $B_{0}$ will be the maximum load pickup. However, the value of load increment typically fluctuates around the forecasted value. In order to make the obtained optimal result robust against the possible errors of the forecasted load increment, the load pickup should be optimally determined so that the actual objective function is robust against the deviation of load increment from its predicted value. It is obvious that a robust decision is reached when the objective function is robust against the maximum fluctuation range of the uncertain load increment. Therefore, the robust optimization model is formulated as, 


$$
\begin{cases}\max \alpha \\ \text { s.t. } & \min B(X, d) \geq B_{\mathrm{m}} \\ & B_{\mathrm{m}}=(1-\delta) B_{0} \\ & H(X, d)=0 \\ & G(X, d) \leq 0 \\ & X \in U(\alpha, \tilde{X})\end{cases}
$$

where $B_{\mathrm{m}}$ is the pre-specified minimum load increment that the new $\mathrm{B}(X, d)$ should not surpass; $B_{0}$ is the optimization result of the deterministic model in (1). This value of $B_{\mathrm{m}}$ can be pre-specified based on the requirements of the decision maker as an input parameter. This value is generally defined as a linear function of $B_{0}$ with the coefficient $\delta$ that indicates the degree of robustness against the value $B_{0}$. The range of coefficient $\delta$ is within the interval $[0,1)$. If the decision maker is risk-averse, a smaller value of $B_{\mathrm{m}}$ can be selected to ensure that $\alpha$ is larger than the actual deviation range. If the decision maker is risk-neutral, a larger value of $B_{\mathrm{m}}$ can be selected to restore more loads with the risk that $\alpha$ may be less than the actual deviation range.

The solution of the above optimization model can obtain a maximum fluctuation range of the uncertain load increment and a load pickup scheme that ensures the amount of load pickup is bigger than the pre-specified minimum load increment. In other words, the solution is robust against unfavorable fluctuation of the uncertain parameter from the forecasted value and satisfies the minimal requirements for system operation.

\subsection{Deterministic load restoration optimization model}

In real-time restoration, the coordination of restoration participants is complicated. The execution of the restoration actions heavily relies on the communication and collaboration among various participants. This paper mainly focuses on the restoration scheme, and assumes that the communication and collaboration among various participants is reliable.

The objective of load restoration is to restore as many important loads as possible. Moreover, the steadystate voltage, power flow and frequency constraints should be satisfied. Accordingly, if the load increment is assumed to be equal to the forecasted increment, the load restoration optimization model is a deterministic model that can be described as follows.

(1) Objective function

Since a large amount of load restoration may result in excessive frequency deviation or severe voltage dip, the restoration process is generally divided into several time steps. Consequently, the optimization objective of load restoration at each time step can be expressed as,

$$
\max f=\sum_{i=1}^{n} \sum_{j=1}^{m_{i}} \omega_{i j} x_{i j} P_{i j}^{L}
$$

where $f$ is the weighted total load pickup, $n$ is the number of load nodes to be restored at each time step, $m_{i}$ is the number of feeder lines at bus $i, \omega_{i j}$ is the weight of the load in the $j$ th feeder line at bus $i, P_{i j}^{L}$ is the forecasted load increment in the $j t h$ feeder line at bus $i$, and $x_{i j}$ is the status of the load bus (this parameter is equal to 1 when the feeder line is resorted; otherwise, its value is 0 ).

(2) Constraints

a) The generator output power constraint: 


$$
\left\{\begin{array}{l}
\sum_{i=1}^{n} \sum_{j=1}^{m_{i}} x_{i j} P_{i j}^{L}<\Delta P_{\Sigma} \\
\Delta P_{\Sigma}=\sum_{i=1}^{N_{G}}\left(P_{i}^{G}(t+\Delta t)-P_{i}^{G}(t)\right)
\end{array}\right.
$$

where $\triangle P_{\Sigma}$ is the total output power increment of generators in one time step, $N_{\mathrm{G}}$ is the number of recovered generators, and $P_{i}^{G}(t)$ is the output power of generator $i$ at time $t$. The model of output power of generator can refer to [4]. The inequality indicates that the maximum load increment must be less than the output power increment of the generators in one time step.

b) The dynamic frequency constraint:

The active power of load increment at one time step must be less than the maximum acceptable active load increment; otherwise, the dynamic frequency may exceed the frequency deviation limit:

$$
P_{\max }^{L} \leq \Delta f_{\max } \sum_{i=1}^{n_{G}} \frac{P_{i}^{N}}{d f_{i}}
$$

where $P_{\max }^{L}$ is the maximum active load increment with respect to the dynamic frequency constraint, $P_{i}^{N}$ is the rated active power of unit $i, \triangle f_{\max }$ is the maximum frequency deviation, and $d f_{i}$ is the dynamic frequency response coefficient of unit $i$. The maximum frequency deviation is set as $0.5 \mathrm{~Hz}$ in this study, and the value of $d f_{i}$ is based on [22].

c) Cold load pickup constraint:

Because the load has cold load pickup characteristic, the active power of load increment at one time step must be less than the maximum active load increment:

$$
P_{i j}^{L} \leq P_{i j}^{C L}(t+\Delta t)-P_{i j}^{C L}(t)
$$

where $P^{C L}$ is the cold load pickup model in [9].

d) The reactive power constraint:

To avoid a voltage over-limit condition caused by the reactive power increments, the reactive load increment must be less than the maximum acceptable reactive load increment:

$$
Q_{i \max }^{L} \leq \frac{\Delta V_{i \max }}{V_{i}^{N}} S_{i}^{s c}
$$

where $Q_{i \max }^{L}$ is the reactive load increment at bus $i$ to be restored at one time step, $\Delta V_{i \max }$ is the maximum voltage deviation of bus $i, V_{i}^{N}$ is the rated voltage of bus $i$, and $S_{i}^{s c}$ is the short-circuit capacity at bus $i$. The maximum voltage deviation is set as $10 \%$ in this study.

e) Power flow constraint:

$$
\left\{\begin{array}{l}
P_{d i}=V_{i} \sum_{j=1}^{N} V_{j}\left(G_{i j} \cos \theta_{i j}+B_{i j} \sin \theta_{i j}\right) \\
Q_{d i}=V_{i} \sum_{j=1}^{N} V_{j}\left(G_{i j} \sin \theta_{i j}-B_{i j} \cos \theta_{i j}\right)
\end{array}\right.
$$

where $P_{d i}$ and $Q_{d i}$ are the injection active and reactive power to bus $i$, respectively; $V_{i}$ is the voltage of bus $i$, $G_{i j}$ and $B_{i j}$ are the conductance and susceptance between bus $i$ and bus $j$, respectively; $\theta_{i j}$ is the phase angle between $V_{i}$ and $V_{j}$; and $N$ is the number of buses. 
f) Operational constraints:

$$
\left\{\begin{array}{l}
P_{i \min }^{G} \leq P_{i}^{G} \leq P_{i \max }^{G} \\
Q_{i \min }^{G} \leq Q_{i}^{G} \leq Q_{i \max }^{G} \\
V_{i \min } \leq V_{i} \leq V_{i \max }
\end{array}\right.
$$

where $P_{i}^{G}$ and $Q_{i}^{G}$ are the active and reactive output power of unit $i$, respectively; $P_{i \max }^{G}$ and $P_{i \min }^{G}$ are the maximum and minimum active power output of unit $i$, respectively; $Q_{i \max }^{G}$ and $Q_{i \min }^{G}$ are the maximum and minimum reactive power output of unit $i$, respectively; $V i$ is the voltage of bus $i$; and $V_{i \max }$ and $V_{i \min }$ are the maximum and minimum voltages of unit $i$, respectively (the maximum and minimum values are set as 0.9 p.u. and 1.1 p.u., respectively, in this study).

In the above model, the restoration of transmission lines and plant auxiliaries can be treated as load restoration for a determined system restoration sequence. Therefore, the topology changes of the transmission line can be neglected in the model.

\subsection{IGDT Model Considering Uncertainty of Load Increment}

The optimization model of (5)-(9) can maximize the load pickup without considering the uncertainty of the load increment $P_{L}$. However, the actual load increment fluctuates around the forecasted load increment. Thus, the optimization model should be transformed by the method in the first subsection into the IGDT-based robust model.

First, the envelope bound model is employed to model the uncertainty of the load increment:

$$
\left\{\begin{array}{l}
P_{i j}^{E} \in U\left(\alpha, P_{i j}^{L}\right) \\
U\left(\alpha, P_{i j}^{E}\right)=\left\{X:\left|\left(P_{i j}^{E}-P_{i j}^{L}\right) / P_{i j}^{L}\right| \leq \alpha\right\}
\end{array}\right.
$$

where $P_{i j}^{E}$ is the actual load increment in the $j$ th feeder line at bus $i$ and the actual load increment fluctuates around its forecasted value within the range of $(1-\alpha, 1+\alpha)$.

To manage the uncertain load increment, the optimization objective is converted to maximize the fluctuation range of uncertain load increment while satisfying the minimum requirement of pre-specified load increment, which can be expressed as,

$$
\begin{gathered}
\max \alpha \\
\sum_{i=1}^{n} \sum_{j=1}^{m_{i}} \omega_{i j} x_{i j} P_{i j}^{E} \geq B_{m} \\
B_{\mathrm{m}}=(1-\delta) B_{0}
\end{gathered}
$$

where $B_{0}$ is the total maximum load pickup based on the deterministic model, and $B_{\mathrm{m}}$ is the pre-specified minimum load increment determined by decision makers according to the historical data or experience.

For a specified $\alpha$, the minimum load increment appears in the lower limit of the uncertain load increment, which is equal to $(1-\alpha) P_{i j}^{L}$. When this increment is larger than the pre-specified minimum load increment, another load increment will satisfy constraint (11.b). Accordingly, (11.b) and (11.c) can be simplified as, 


$$
\left\{\begin{array}{c}
\sum_{i=1}^{n} \sum_{j=1}^{m_{i}} \omega_{i j} x_{i j}(1-\alpha) P_{i j}^{L} \geq B_{m} \\
B_{m}=(1-\delta) B_{0}
\end{array}\right.
$$

Similarly, for a specified $\alpha$, the maximum load increment appears in the upper limit of uncertain load increment, which is equal to $(1+\alpha) P_{i j}^{L}$. When this increment satisfies the generator output power constraint, other load increment will satisfy the constraint. Equation (5) can be modified as,

$$
\left\{\begin{array}{c}
\sum_{i=1}^{n} \sum_{j=1}^{m_{i}} x_{i j}(1+\alpha) P_{i j}^{L}<\Delta P_{\Sigma} \\
\Delta P_{\Sigma}=\sum_{i=1}^{N_{G}}\left(P_{i}^{G}(t+\Delta t)-P_{i}^{G}(t)\right)
\end{array}\right.
$$

Moreover, when the upper limit of the uncertain load increment satisfies the dynamic frequency constraint, another load increment will be less than the maximum acceptable active load increment. Thus, (6) and (7) can be modified as,

$$
\begin{gathered}
(1+\alpha) x_{i j} P_{i j}^{L} \leq \Delta f_{\max } \sum_{i=1}^{n_{G}} \frac{P_{i}^{N}}{d f_{i}} \\
(1+\alpha) x_{i j} P_{i j}^{L} \leq P_{i j}^{C L}(t+\Delta t)-P_{i j}^{C L}(t)
\end{gathered}
$$

Similarly, when the upper limit of the uncertain load increment satisfies the reactive power constraint, another load increment will be less than the maximum acceptable reactive load increment. Thus, (7) can be modified as follows:

$$
(1+\alpha) x_{i j} Q_{i j}^{L} \leq \frac{\Delta V_{i \max }}{V_{i}^{N}} S_{i}^{s c}
$$

where $Q_{\mathrm{L} i j}$ is the forecasted reactive power in the $j t h$ feeder line at bus $i$.

Furthermore, for the power flow constraint, when the upper and lower limits of the uncertain load increment make the power flow converge, another load increment will satisfy the constraint. In addition, when the upper and lower limits of the uncertain load increment satisfy the operational constraint, other load increment will continue to satisfy the constraint. Therefore, the power flow constraint (8) can be expressed as,

$$
\begin{aligned}
& \left\{\begin{array}{l}
P_{i}^{G}-(1-\alpha) P_{i}^{L}=V_{i} \sum_{j=1}^{N} V_{j}\left(G_{i j} \cos \delta_{i j}+B_{i j} \sin \delta_{i j}\right) \\
Q_{i}^{G}-(1-\alpha) Q_{i}^{L}=V_{i} \sum_{j=1}^{N} V_{j}\left(G_{i j} \sin \delta_{i j}-B_{i j} \cos \delta_{i j}\right)
\end{array}\right. \\
& \left\{\begin{array}{l}
P_{i}^{G}-(1+\alpha) P_{i}^{L}=V_{i} \sum_{j=1}^{N} V_{j}\left(G_{i j} \cos \delta_{i j}+B_{i j} \sin \delta_{i j}\right) \\
Q_{i}^{G}-(1+\alpha) Q_{i}^{L}=V_{i} \sum_{j=1}^{N} V_{j}\left(G_{i j} \sin \delta_{i j}-B_{i j} \cos \delta_{i j}\right)
\end{array}\right.
\end{aligned}
$$

where $P_{\mathrm{L} 0 \_i}$ and $Q_{\mathrm{L} 0 \_i}$ are the previously restored active and reactive loads at bus $i$. 
In summary, the robust optimization model considering the uncertainty load increment can be expressed as,

$$
\left\{\begin{array}{l}
\max \alpha \\
0 \leq \alpha \leq 1 \\
\text { s.t. }(10),(13) \sim(19)
\end{array}\right.
$$

A robust load restoration scheme obtained using the model is robust against unfavorable deviations of the load increment from the forecasted value. In other words, the solution guarantees that the weighted load pickup is not worse than $(1-\delta) B_{0}$ if the actual load increment fluctuates around its forecasted value within the range of $(1-\alpha, 1+\alpha)$.

\section{Model Transformation and Solution Method}

The robust optimization model based on the IGDT in Section II is a nonlinear model, which is challenging to solve efficiently. In this section, the model is transformed to an SOCP model.

\subsection{Power Flow Equation Relaxation}

The robust optimization model is a nonlinear model due to the nonlinear equations (12)-(17) that result from the multiplying of continuous variables by $0-1$ variables, and the nonlinearity of (16) results from the multiplying of continuous variables by continuous variables. The AC power flow equation (16) is challenging to linearize.

The DC power flow is usually employed to replace AC power flow to accelerate solution speed [23]. However, the bus voltage in the DC power flow model is fixed, which decreases the solution accuracy. Consequently, it is not suitable for the problem in this paper. The SOCP relaxation has been proven to be exact for radial networks with some conditions [24-25]. For some special cases, in which the convex relaxation is generally inexact, the concave convex procedure (CCP) [26] is combined with the SOCP to form a sequential SOCP method to enhance the solution ability [29]. During system restoration, the restoration process is restoring the non-black-start units in sequence. The network of reenergized system is a radial network [4] which can be relaxed by the SOCP. Therefore, the SOCP relaxation is employed to relax the power flow constraint in this paper.

Because the SOCP relaxation model is based on the branch flow model, the branch flow equations are used to describe the power flow constraint. The branch flow equations are still nonlinear equations but can be relaxed via the cone relaxation technique and transformed into an SOCP model [25]. Therefore, in this subsection, the power flow equation is transformed through the branch flow equation into an SOCP model.

(1) Branch Flow Equations

Based on the relationships between the impedance of a line, the voltages at two ends of a line, the current of a line, and the bus power injection, the branch flow model can be expressed as,

$$
\begin{gathered}
P_{j}^{G}-P_{j}^{L}=\sum_{k: j \rightarrow k} P_{j k}-\sum_{i: i \rightarrow j}\left(P_{i j}-r_{i j} l_{i j}\right)+g_{j}\left|V_{j}\right|^{2} \\
Q_{j}^{G}-Q_{j}^{L}=\sum_{k: j \rightarrow k} Q_{j k}-\sum_{i: i \rightarrow j}\left(Q_{i j}-x_{i j} l_{i j}\right)+b_{j}\left|V_{j}\right|^{2} \\
\left|V_{j}\right|^{2}=\left|V_{i}\right|^{2}-2\left(r_{i j} P_{i j}+x_{i j} Q_{i j}\right)+\left(r_{i j}^{2}+x_{i j}^{2}\right) l_{i j}
\end{gathered}
$$




$$
l_{i j}=\frac{P_{i j}^{2}+Q_{i j}^{2}}{\left|V_{i}\right|^{2}}
$$

where $r_{i j}$ and $x_{i j}$ are the resistance and reactance of a line, respectively; $g_{i}$ and $b_{i}$ are the conductance and susceptance from bus $i$ to the ground, respectively; $i \rightarrow j$ denotes a line pointing from node $i$ to node $j ; P_{i j}$ and $Q_{i j}$ are the sending-end active and reactive power from bus $i$ to bus $j$, respectively; and $V_{i}$ is the complex voltage in bus $i ; l_{i j}$ are the square of current.

(2) Convex Relaxations of the Branch Flow Equations

The SOCP is an extension of linear programming, and the SOCP problem is a convex programming problem [28] that can be solved in polynomial time via the interior point method. In general, a conic optimization problem can be expressed in the following form [29],

$$
\min _{x_{i}}\left\{c^{T} x \mid A x=b, x_{i} \in K, i=1,2, \mathrm{~K}, N\right\}
$$

where variable $x \in R_{N}$; coefficient constants $b \in R_{M}, c \in R_{N}$, and $A_{M \times N} \in R_{M \times N}$; and $K$ is assumed to be a pointed closed convex cone that can be expressed as,

$$
K=\left\{x_{i} \in R_{N} \mid y^{2} \geq \sum_{i=1}^{N} x_{i}^{2}, y \geq 0\right\}
$$

or

$$
K=\left\{x_{i} \in R_{N} \mid y z \geq \sum_{i=1}^{N} x_{i}^{2}, y, z \geq 0\right\}
$$

The quadratic inequalities in (24) and (25) are the second-order cone constraint and rotated second-order cone constraint, respectively.

Since (22) in the branch flow equations is a non-convex quadratic equality, which is challenging to solve, it is relaxed to a rotated second-order cone constraint:

$$
l_{i j} \geq \frac{P_{i j}^{2}+Q_{i j}^{2}}{v_{i}}, \forall(i, j) \in E
$$

Moreover, with $V_{i}^{2}$ replaced by $v_{i}$, (26) can be written as the following standard second-order cone constraint:

where \|\|$_{2}$ is the Frobenius norm.

$$
\left\|\begin{array}{c}
2 P_{i j} \\
2 Q_{i j} \\
l_{i j}-v_{i}
\end{array}\right\|_{2} \leq l_{i j}+v_{i}
$$

\subsection{Mixed-Integer SOCP Model for Load Restoration}

In addition to the power flow constraint in the robust model of load restoration, the constraints (12)-(15) must be linearized due to the addition of uncertainty $\alpha$.

A new variable $m_{i j}$ is introduced whose value is the multiplication of the 0-1 variable $x_{i j}$ and continuous variable $\alpha$. When $x_{i j}$ is $0, m_{i j}$ is 0 , and $x_{i j}$ is $1, m_{i j}$ is equal to $\alpha$. In addition, the range of continuous variable $\alpha$ is 
$[0,1]$. Therefore, $m_{i j}=x_{i j} \times \alpha$ can be equivalent to the constraints given below:

$$
\begin{gathered}
0 \leq \alpha \leq 1 \\
0 \leq m_{i j} \leq x_{i j} \\
\alpha+x_{i j}-1 \leq m_{i j} \leq \alpha-x_{i j}+1
\end{gathered}
$$

Based on the above constraints, (12)-(15) can be linearized as,

$$
\begin{gathered}
\left\{\begin{array}{c}
\sum_{i=1}^{n} \sum_{j=1}^{m_{i}} \omega_{i j}\left(x_{i j}-m_{i j}\right) P_{i j}^{L} \geq B_{m} \\
B_{m}=(1-\delta) B_{0}
\end{array}\right. \\
\left\{\begin{array}{c}
\sum_{i=1}^{n} \sum_{j=1}^{m}\left(x_{i j}+m_{i j}\right) P_{i j}^{L}<\Delta P_{\Sigma} \\
\Delta P_{\Sigma}=\sum_{i=1}^{N_{G}}\left(P_{i}^{G}(t+\Delta t)-P_{i}^{G}(t)\right)
\end{array}\right. \\
\left(x_{i j}+m_{i j}\right) P_{i j}^{L} \leq \Delta f_{\max } \sum_{i=1}^{n_{G}} \frac{P_{i}^{N}}{d f_{i}} \\
\left(x_{i j}+m_{i j}\right) P_{i j}^{L} \leq P_{i j}^{C L}(t+\Delta t)-P_{i j}^{C L}(t) \\
\left(x_{i j}+m_{i j}\right) Q_{i j}^{L} \leq \frac{\Delta U_{i m \mathrm{ax}}}{U_{i N}} S_{i}^{s c}
\end{gathered}
$$

Based on the convex relaxation and linearization above, all nonlinear constraints are transformed into second-order cone constraints and linear constraints. A mixed-integer SOCP of load restoration is formulated as,

$$
\left\{\begin{array}{l}
\max \alpha \\
\text { s.t. }(10),(21) \sim(23),(29) \sim(37)
\end{array}\right.
$$

\subsection{Solution Method}

The optimization problems in a power system are generally modeled as a mixed integer programming model to be solved using business solver CPLEX, which can ensure the accuracy of solution and also offers significant calculation efficiency. The proposed model in (35) is a mixed-integer SOCP problem which can be solved by CPLEX.

\section{Case Study}

The simulations in this paper were executed on a PC with Intel CoreTM i5 CPU @3.10 GHz and 8 GB RAM. The proposed mixed-integer SOCP model was solved using IBM ILOG CPLEX 12.4. The New England 10-machine 39-bus test system in Fig. 1 is employed to validate the effectiveness of the proposed method. Suppose the system has a blackout, unit \#30 is a black start unit with self-restarting ability, and the other units are thermal generators without restarting ability during restoration. 
Assume that units \#37, \#38, \#39, and \#33, in addition to the black start unit, have been restored and that unit \#34 is waiting for restart at this time step. The blue solid lines in Fig. 1 are the energized lines. The output power of units \#37, \#38, and \#39 is $153.6 \mathrm{MW}, 167.4 \mathrm{MW}$, and $99 \mathrm{MW}$, respectively, and unit \#33 has been restored but is still in the warm-up stage. The restored loads at bus 25, bus 26, bus 29, bus 39, bus 27, and bus 16 are $76 \mathrm{MW}, 40 \mathrm{MW}, 3 \mathrm{MW}, 134 \mathrm{MW}, 128 \mathrm{MW}$, and $64 \mathrm{MW}$, respectively. The cranking power of unit \#34 is provided by the restored system, and the restoration path is 19-20-34 as shown by the red solid lines in Fig. 1. To reduce the additional restoration time, only the loads on the restoration path and the restored system can be restored, i.e., the loads at buses 16, 20, 27, 25, 26, 29, and 39. The components of each load bus and their load amounts and weights are listed in Table 1. The importance of a load, which is the proportion of Class-I load in this study [30], is expressed as its weight; loads with larger weight are prioritized during restoration.

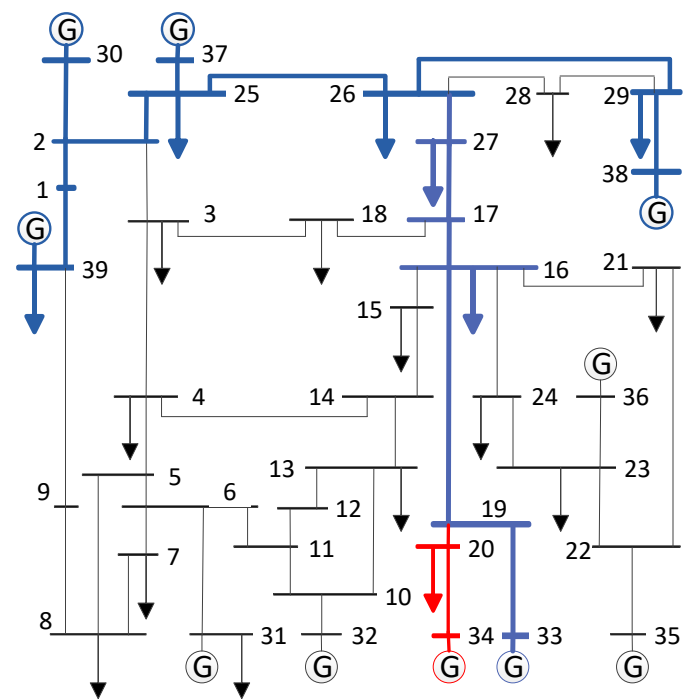

Fig. 1 IEEE-39 BUS SYSTEM

Table 1: The load parameter in next time step

\begin{tabular}{lc}
\hline Bus & $\begin{array}{c}\text { Forecasted load of each feeder line in different bus (MW) } \\
\text { /weight }\end{array}$ \\
\hline 25 & $16 / 0.31,32 / 0.15,100 / 0.33$ \\
26 & $10 / 0.14,14 / 0.25,15 / 0.23,20 / 0.4,40 / 0.3$ \\
29 & $13 / 0.1,100 / 0.21,90 / 0.3,35 / 0.27,42.5 / 0.29$ \\
39 & $40 / 0.2,60 / 0.32,60 / 0.14,80 / 0.6,80 / 0.12$, \\
27 & $100 / 0.4,100 / 0.8,150 / 0.3,150 / 0.58,150 / 0.44$ \\
16 & $6 / 0.65,80 / 0.2,59 / 0.28,60 / 0.4$ \\
20 & $18 / 0.33,22 / 0.25,36 / 0.43,36 / 0.28,63 / 0.38,90 / 0.6$ \\
& $25 / 0.75,25 / 0.36,35 / 0.26,35 / 0.54,40 / 0.48,50 / 0.29$, \\
& $50 / 0.55,60 / 0.44,60 / 0.46,80 / 0.38,100 / 0.82,120 / 0.5$ \\
\hline
\end{tabular}




\subsection{Optimization Results of the Load Restoration Based on IGDT}

If the load increment uncertainty is not considered at the time step, the optimal scheme is restoring the 1st feeder line of bus 26, the 4 th feeder line of bus 39 and the 1 st feeder line of bus 27 . When taking into account the load uncertainty, the amount of load pickup will be less than the results of deterministic model. The maximum fluctuation range of uncertainty $\alpha$ is related to the pre-specified minimum load increments, which are determined by the deviation factor $\delta$. In this case, the relationship between the maximum fluctuation range of the uncertainty and deviation factor is shown in Table 2 and Fig. 2.

Fig. 2 and Table 2 indicate that the relationship between $\alpha$ and $\delta$ is positively correlated. With an increase in $\delta$, the maximum fluctuation range of uncertainty $\alpha$ also increases, i.e., a higher range of load forecast errors can be accommodated at the cost of a lower load pickup. In other words, a lower load pickup is correlated with improved robustness of the corresponding load restoration scheme. The restoration scheme can be selected by dispatchers with respect to the fluctuation range based on historical data.

Table 2: Robust optimization results of the load restoration based on IGDT

\begin{tabular}{ccccc}
\hline$\delta$ & $\alpha$ & Restored loads & $\begin{array}{r}\text { Optimization } \\
\text { result }\end{array}$ & $\begin{array}{r}\text { Prespecified minimum load } \\
\text { increment }\end{array}$ \\
\hline 0.1 & 0.08 & $39(4), 27(1)$ & 47.97 & 47.97 \\
0.2 & 0.13 & $39(4), 27(1)$ & 44.98 & 42.64 \\
0.3 & 0.22 & $39(4)$ & 37.52 & 37.31 \\
0.4 & 0.22 & $39(4)$ & 37.52 & 31.98 \\
0.5 & 0.22 & $39(4)$ & 37.52 & 26.65 \\
0.6 & 0.22 & $39(4)$ & 37.52 & 21.32 \\
0.7 & 0.31 & $39(2), 27(1)$ & 15.99 & 15.99 \\
0.8 & 0.50 & $25(1), 29(5), 27(1)$ & 10.66 & 10.66 \\
0.9 & 0.71 & $25(1), 29(4), 27(1)$ & 5.33 & 5.33 \\
\hline
\end{tabular}

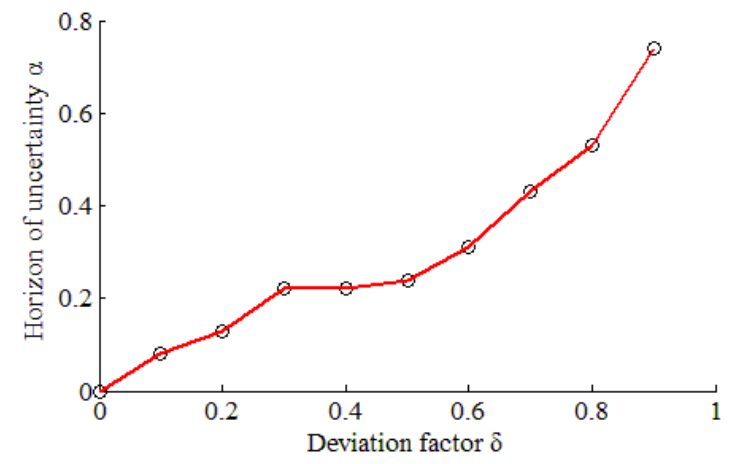

Fig. 2 The relationship between $\alpha$ and $\delta$ when restoring unit \#34

\subsection{Comparison of IGDT with other methods}

To validate the effectiveness of the IGDT, the results of the IGDT, deterministic model, fuzzy chance constrained model are compared in this subsection. In the fuzzy chance constrained model, the confidence level and risk coefficient of the model are set as 0.9 and 0.2, respectively. Because fuzzy parameters of load 
increment are hard to obtain, 5 experimental groups of parameters listed in Table 3 are chosen to select the best fuzzy parameters. The fuzzy parameters of group 5 are the most conservative, having the lowest weighted load amount, so these are selected for simulation.

Table 3: Optimal results for different fuzzy parameters

\begin{tabular}{|c|c|c|c|c|c|c|}
\hline \multicolumn{2}{|c|}{ Group Number } & 1 & 2 & 3 & 4 & 5 \\
\hline \multirow{6}{*}{ Fuzzy parameter } & $\beta$ & 0.9 & 0.9 & 0.9 & 0.9 & 0.9 \\
\hline & $\lambda$ & 0.2 & 0.2 & 0.2 & 0.2 & 0.2 \\
\hline & $\mu 1$ & 0.5 & 0.6 & 0.7 & 0.5 & 0.5 \\
\hline & $\mu 2$ & 0.6 & 0.9 & 0.8 & 0.6 & 0.7 \\
\hline & $\mu 3$ & 1 & 1.2 & 0.9 & 0.7 & 1.1 \\
\hline & $\mu 4$ & 1.1 & 1.3 & 1 & 0.8 & 1.3 \\
\hline \multicolumn{2}{|c|}{ Weighted load amount } & 51.9 & 20.4 & 20.4 & 86.16 & 16.86 \\
\hline
\end{tabular}

Table 4: Comparison between the Restoration Amounts based on Three Different Models

\begin{tabular}{|c|c|c|c|c|c|c|c|}
\hline Scenario & $\begin{array}{c}\text { Fluctuation } \\
\text { of the load } \\
\text { amount }\end{array}$ & $\begin{array}{c}\text { Weighted } \\
\text { load pickup } \\
\text { based on } \\
\text { model } 1\end{array}$ & $\begin{array}{c}\text { Problems of } \\
\text { Model } 1\end{array}$ & $\begin{array}{c}\text { Weighted } \\
\text { load pickup } \\
\text { based on } \\
\text { model } 2\end{array}$ & $\begin{array}{c}\text { Problems of } \\
\text { Model } 2\end{array}$ & $\begin{array}{c}\text { Weighted } \\
\text { load pickup } \\
\text { based on } \\
\text { model } 3 \\
\end{array}$ & $\begin{array}{c}\text { Problems } \\
\text { of Model } \\
3\end{array}$ \\
\hline 1 & 0.71 & 11.97 & 1 & 37.84 & 1 & 16.40 & 1 \\
\hline 2 & 1.28 & 0 & $\begin{array}{c}\text { Voltage } \\
\text { violation at } \\
\text { bus } 34\end{array}$ & 0 & $\begin{array}{l}\text { System } \\
\text { frequency } \\
\text { violation }\end{array}$ & 29.57 & l \\
\hline
\end{tabular}

Suppose that the fluctuation range of the load increment centered at the forecasted value is $[0.7,1.3]$ and the actual load increment is randomly generated within this range for each simulation. The simulation is repeated 20 times for analysis; the weighted amounts of load pickup of the three different models are shown in Fig. 3. Note that if the load restoration scheme cannot satisfy the security constraints due to the fluctuation of the load increment, the weighted restoration amount will be set as 0 .

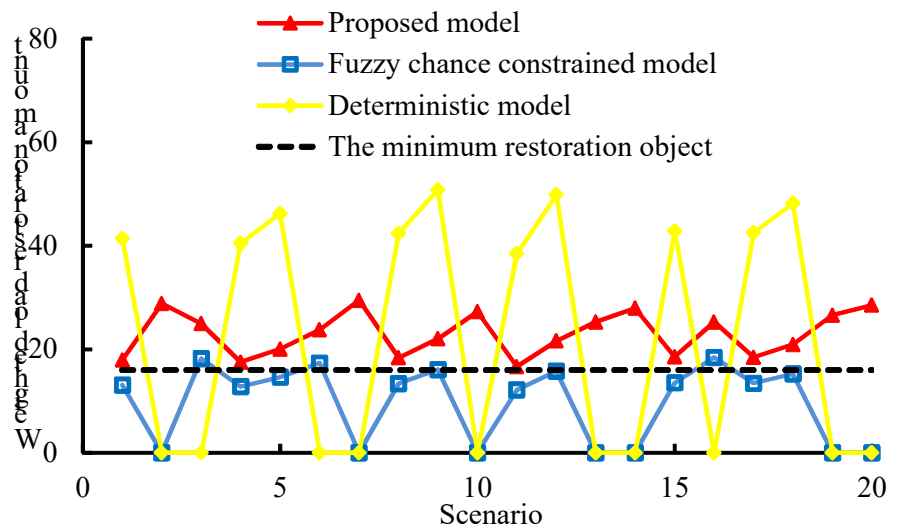

Fig.3. Comparison among the simulation results based on three different models

Fig. 3 shows that the restoration schemes of the fuzzy chance constrained model or deterministic model cannot always satisfy the security constraint because of the uncertainty of the load increment. As a result, the restoration process of the power system will be suspended. Three of these results violating the security 
constraints are specified in Table 4.

Model 1 in Table 4 is a fuzzy chance constrained model of load restoration, Model 2 represents the deterministic model of load restoration, and Model 3 represents the robust model proposed. When the load increment fluctuates in the range $[0.7,1.3]$ around the forecasted value, the load restoration schemes by the three models are different. The restoration scheme determined by the fuzzy chance constrained model involves restoring the 1st feeder line of bus 25 and 4th feeder line of bus 26 and 1 st feeder line of bus 27; the restoration scheme determined by the deterministic model is restoring the 1 st feeder line of bus 26 , 4 th feeder line of bus 39 and 1st feeder line of bus 27; and the last restoration scheme determined by the proposed model is restoring the 2 nd feeder line of bus 39 and 1 st feeder line of bus 27 .

The actual load increment of Scenario 1 in Table 4 is 0.71 times the forecasted value. The restoration schemes of all three models can satisfy the security constraints. In the schemes, the optimal result of the proposed model is more conservative with less load pickup, but the actual load increment is larger than the pre-specified minimum load increments. In Scenario 2, the actual load pickup is 1.28 times the forecasted value. The restoration schemes by the fuzzy chance constrained model and deterministic model both result in power shortage because of the excessive load pickup that leads to the frequency over-limit condition. In addition, the restoration scheme determined by the fuzzy chance constrained model also results in voltage violation; specifically, the voltage of buses 33 and 34 are 0.897 and 0.894 , respectively, none of which satisfies the constraints.

Figure 3 and Table 4 show that the robust load restoration model proposed can endure the load fluctuation within an allowable range, and the adverse effects caused by the uncertainty of load amount can be effectively avoided. As long as an appropriate load restoration scheme is selected, the minimum restoration object can be achieved without compromising security. Although the load restoration amount is smaller, the stability and security of the restoration process can be guaranteed.

\subsection{The Exactness of Convex Relaxations}

In order to confirm the exactness of the proposed SOCP model, the results with the proposed model are compared with the results of the original nonlinear model for different restoration scenarios. Because the nonlinear model is hard to solve, the Artificial Bee Colony (ABC) Algorithm is employed to solve the nonlinear model. The simulation parameters of the $\mathrm{ABC}$ are set as follows. The number of the population $\mathrm{N}=20$, the maximum number of iterations $\mathrm{MCN}=200$, and the maximum number of exploitations Limit $=5$.

The load restoration results with the original nonlinear model, linear model and proposed SCOP model for restoring the generator at Bus 37, Bus 38 and Bus 34 are illustrated in Tables 5-7. Most of the optimization results of the original nonlinear model and proposed SCOP model are the same. When the deviation factors are $0.3,0.5,0.7,0.8$ and 0.9 in Table 6 , the gap values between the proposed SOCP model and the original nonlinear model are $0.25,0.05,0.03,0.23$, and 0.18 respectively. When the deviation factors are $0.7,0.8$ and 0.9 in Table 7 , the gap values are $0.08,0.08$, and 0.19 respectively. The optimization results of the nonlinear model are all worse than the results of the SOCP model because the ABC algorithm is a heuristic method which may be trapped in the local optimal solution. Furthermore, when restoring Bus 37, only the load at Bus 25 can be restored, and it is easy to obtain the optimal result. Both of the two models obtain the optimal result. Therefore, the SOCP model can obtain better results than the ABC algorithm under this paper's cases. 
Table 5: Optimization results of the load restoration for the time step restoring the generator at Bus 37

\begin{tabular}{|c|c|c|c|c|c|c|}
\hline$\delta$ & $\begin{array}{l}\text { Pre-specified } \\
\text { mini. load } \\
\text { increment }\end{array}$ & model & $\alpha$ & Restored loads & $\begin{array}{l}\text { Optimization } \\
\text { result }\end{array}$ & $\begin{array}{l}\text { Gap value between } \\
\text { SOCP model and } \\
\text { original nonlinear model }\end{array}$ \\
\hline \multirow{2}{*}{0.1} & \multirow{2}{*}{14.94} & nonlinear & 0.100 & $25(1)(3)$ & 14.94 & \multirow{2}{*}{0} \\
\hline & & SOCP & 0.100 & $25(1)(3)$ & 14.94 & \\
\hline \multirow{2}{*}{0.2} & \multirow{2}{*}{13.28} & nonlinear & 0.200 & $25(1)(3)$ & 13.28 & \multirow{2}{*}{0} \\
\hline & & SOCP & 0.200 & $25(1)(3)$ & 13.28 & \\
\hline \multirow{2}{*}{0.3} & \multirow{2}{*}{11.62} & nonlinear & 0.250 & $25(1)(3)$ & 12.45 & \multirow{2}{*}{0} \\
\hline & & SOCP & 0.250 & $25(1)(3)$ & 12.45 & \\
\hline \multirow{2}{*}{0.4} & \multirow{2}{*}{9.96} & nonlinear & 0.250 & $25(1)(3)$ & 12.45 & \multirow{2}{*}{0} \\
\hline & & SOCP & 0.250 & $25(1)(3)$ & 12.45 & \\
\hline \multirow{2}{*}{0.5} & \multirow{2}{*}{8.30} & nonlinear & 0.282 & $25(1)(2)$ & 8.30 & \multirow{2}{*}{0} \\
\hline & & SOCP & 0.282 & $25(1)(2)$ & 8.30 & \\
\hline \multirow{2}{*}{0.6} & \multirow{2}{*}{6.64} & nonlinear & 0.425 & $25(1)(2)$ & 6.64 & \multirow{2}{*}{0} \\
\hline & & SOCP & 0.425 & $25(1)(2)$ & 6.64 & \\
\hline \multirow{2}{*}{0.7} & \multirow{2}{*}{4.98} & nonlinear & 0.569 & $25(1)(2)$ & 5.02 & \multirow{2}{*}{0} \\
\hline & & SOCP & 0.569 & $25(1)(2)$ & 5.02 & \\
\hline \multirow{2}{*}{0.8} & \multirow{2}{*}{3.32} & nonlinear & 0.667 & $25(1)(2)$ & 3.85 & \multirow{2}{*}{0} \\
\hline & & SOCP & 0.667 & $25(1)(2)$ & 3.85 & \\
\hline \multirow{2}{*}{0.9} & \multirow{2}{*}{1.66} & nonlinear & 0.748 & $25(1)$ & 1.664 & \multirow{2}{*}{0} \\
\hline & & SOCP & 0.748 & $25(1)$ & 1.664 & \\
\hline
\end{tabular}

Table 6: Optimization results of the load restoration for the time step restoring the generator at Bus 38

\begin{tabular}{|c|c|c|c|c|c|c|}
\hline$\delta$ & $\begin{array}{l}\text { Pre-specified } \\
\text { mini. load } \\
\text { increment }\end{array}$ & model & $\alpha$ & Restored loads & $\begin{array}{l}\text { Optimization } \\
\text { result }\end{array}$ & $\begin{array}{c}\text { Gap value between } \\
\text { SOCP model and } \\
\text { original nonlinear } \\
\text { model }\end{array}$ \\
\hline \multirow{2}{*}{0.1} & \multirow{2}{*}{20.35} & nonlinear & 0.10 & $26(1)(2)(3)(4)(5), 29(1)$ & 20.35 & \multirow{2}{*}{0} \\
\hline & & SOCP & 0.10 & $26(1)(2)(3)(4)(5), 29(1)$ & 20.35 & \\
\hline \multirow{2}{*}{0.2} & \multirow{2}{*}{18.09} & nonlinear & 0.13 & $26(1)(2)(3)(4)(5)$ & 18.09 & \multirow{2}{*}{0} \\
\hline & & SOCP & 0.13 & $26(1)(2)(3)(4)(5)$ & 18.09 & \\
\hline \multirow{2}{*}{0.3} & \multirow{2}{*}{15.83} & nonlinear & 0.22 & $26(2)(3)(4)(5), 29(1)$ & 16.08 & \multirow{2}{*}{0.25} \\
\hline & & SOCP & 0.23 & $26(2)(3)(4)(5), 29(1)$ & 15.83 & \\
\hline \multirow{2}{*}{0.4} & \multirow{2}{*}{13.57} & nonlinear & 0.29 & $26(1)(2)(4)(5), 29(1)$ & 13.66 & \multirow{2}{*}{0} \\
\hline & & SOCP & 0.29 & $26(1)(2)(4)(5), 29(1)$ & 13.66 & \\
\hline \multirow{2}{*}{0.5} & \multirow{2}{*}{11.31} & nonlinear & 0.35 & $26(2)(3)(4), 29(1)$ & 11.92 & \multirow{2}{*}{0.05} \\
\hline & & SOCP & 0.36 & $26(2)(3)(4)(5)$ & 11.87 & \\
\hline \multirow{2}{*}{0.6} & \multirow{2}{*}{9.04} & nonlinear & 0.48 & $26(2)(4)(5), 29(1)$ & 9.04 & \multirow{2}{*}{0} \\
\hline & & SOCP & 0.48 & $26(2)(4)(5), 29(1)$ & 9.04 & \\
\hline \multirow{2}{*}{0.7} & \multirow{2}{*}{6.78} & nonlinear & 0.56 & $29(1)(2)(3)(5), 29(1)$ & 6.81 & \multirow{2}{*}{0.03} \\
\hline & & SOCP & 0.57 & $26(1)(2)(3)(5), 29(1)$ & 6.78 & \\
\hline \multirow{2}{*}{0.8} & \multirow{2}{*}{4.52} & nonlinear & 0.69 & $26(2)(3)(4), 29(1)$ & 4.78 & \multirow{2}{*}{0.23} \\
\hline & & SOCP & 0.71 & $26(2)(3)(4), 29(1)$ & 4.55 & \\
\hline 0.9 & 2.26 & nonlinear & 0.82 & $26(2)(3)(4)$ & 2.44 & 0.18 \\
\hline
\end{tabular}


$\begin{array}{llll}\text { SOCP } & 0.83 & 26(1)(2)(3)(5) & 2.26\end{array}$

Table 7: Optimization results of the load restoration for the time step restoring the generator at Bus 34

\begin{tabular}{|c|c|c|c|c|c|c|}
\hline$\delta$ & $\begin{array}{l}\text { Pre-specified } \\
\text { mini. load } \\
\text { increment }\end{array}$ & model & $\alpha$ & Restored loads & $\begin{array}{l}\text { Optimization } \\
\text { result }\end{array}$ & $\begin{array}{c}\text { Gap value between } \\
\text { SOCP model and original } \\
\text { nonlinear model }\end{array}$ \\
\hline \multirow{2}{*}{0.1} & \multirow{2}{*}{47.97} & nonlinear & 0.08 & $39(4), 27(1)$ & 47.97 & \multirow{2}{*}{0} \\
\hline & & SOCP & 0.08 & $39(4), 27(1)$ & 47.97 & \\
\hline \multirow{2}{*}{0.2} & \multirow{2}{*}{42.64} & nonlinear & 0.13 & $39(4), 27(1)$ & 44.98 & \multirow{2}{*}{0} \\
\hline & & SOCP & 0.13 & $39(4), 27(1)$ & 44.98 & \\
\hline \multirow{2}{*}{0.3} & \multirow{2}{*}{37.31} & nonlinear & 0.22 & $39(4)$ & 37.52 & \multirow{2}{*}{0} \\
\hline & & SOCP & 0.22 & $39(4)$ & 37.52 & \\
\hline \multirow{2}{*}{0.4} & \multirow{2}{*}{31.98} & nonlinear & 0.22 & $39(4)$ & 37.52 & \multirow{2}{*}{0} \\
\hline & & SOCP & 0.22 & $39(4)$ & 37.52 & \\
\hline \multirow{2}{*}{0.5} & \multirow{2}{*}{26.65} & nonlinear & 0.22 & $39(4)$ & 37.52 & \multirow{2}{*}{0} \\
\hline & & SOCP & 0.22 & $39(4)$ & 37.52 & \\
\hline \multirow{2}{*}{0.6} & \multirow{2}{*}{21.32} & nonlinear & 0.22 & $39(4)$ & 37.52 & \multirow{2}{*}{0} \\
\hline & & SOCP & 0.22 & $39(4)$ & 37.52 & \\
\hline \multirow{2}{*}{0.7} & \multirow{2}{*}{15.99} & nonlinear & 0.31 & $39(2), 27(1)$ & 16.07 & \multirow{2}{*}{0.08} \\
\hline & & SOCP & 0.31 & $39(2), 27(1)$ & 15.99 & \\
\hline \multirow{2}{*}{0.8} & \multirow{2}{*}{10.66} & nonlinear & 0.50 & $25(1), 29(5), 27(1)$ & 10.78 & \multirow{2}{*}{0.08} \\
\hline & & SOCP & 0.50 & $25(1), 29(5), 27(1)$ & 10.66 & \\
\hline \multirow{2}{*}{0.9} & \multirow{2}{*}{5.33} & nonlinear & 0.70 & $25(1), 29(4), 27(1)$ & 5.52 & \multirow{2}{*}{0.19} \\
\hline & & SOCP & 0.71 & $25(1), 29(4), 27(1)$ & 5.33 & \\
\hline
\end{tabular}

The results in Tables 5-7 can also demonstrate the restoration process. For the time step in Table 5, few loads can be integrated to the restored system. With the restoration of the power system, the loads which are allowed to integrate into the restored system are increased significantly at the time step in Table7. For the results in each time step, the relationship between $\alpha$ and $\delta$ is positively correlated as shown in Fig. 2.

\section{Conclusion}

Load restoration plays an important role in the power system restoration. Since the uncertainty of the load increment can threaten the security of power system restoration, a load restoration optimization model is proposed considering the uncertainty of the load increment based on the IGDT. This model is transformed into a mixed-integer SOCP model. The simulation results demonstrate that the solution method for the SOCP model has similar accuracy as the commercial BPA software. Furthermore, the robust load restoration scheme obtained based on the proposed method satisfies the security constraints and achieve the pre-specified minimum load increment rapidly and accurately. The model and solution method can be used by system dispatchers to determine the load restoration plan after a blackout.

The SOCP model in this paper is suitable for the radial network. However, the network will be a meshed network at the end of load restoration. The convex optimization for meshed networks needs to be studied in the future research. 


\section{References}

[1] Xue Y and Xiao S. Generalized congestion of power systems: insights from the massive blackouts in India[J]. Journal of Modern Power Systems and Clean Energy, 2013, 1(2): 91-100.

[2] Zhang Q, Ishihara K N, Mclellan B C, et al. Scenario analysis on future electricity supply and demand in Japan[J]. Energy, 2012, 38(1): 376-385.

[3] Wu F F, Moslehi K and Bose A. Power system control centers: Past, present, and future[J]. Proceedings of the IEEE, 2005, 93(11): 1890-1908.

[4] Sun W, Liu C C and Zhang L. Optimal generator start-up strategy for bulk power system restoration[J]. IEEE Transactions on Power Systems, 2011, 26(3): 1357-1366.

[5] Qu H and Liu Y. Maximizing restorable load amount for specific substation during system restoration[J]. International Journal of Electrical Power \& Energy Systems, 2012, 43(1): 1213-1220.

[6] Qin Z, Hou Y, Liu C C, et al. Coordinating generation and load pickup during load restoration with discrete load increments and reserve constraints[J]. IET Generation, Transmission \& Distribution, 2015, 9(15): 2437-2446.

[7] Agneholm E and Daalder J. Load recovery in different industries following an outage[J]. IEE Proceedings-Generation, Transmission and Distribution, 2002, 149(1): 76-82.

[8] Mirza O H. Usage of CLPU curve to deal with the cold load pickup problem[J]. IEEE Transactions on power delivery, 1997, 12(2): 660-667.

[9] Medina D R, Rappold E, Sanchez O, et al. Fast assessment of frequency response of cold load pickup in power system restoration[J]. IEEE Transactions on Power Systems, 2016, 31(4). 3249-3256.

[10] Kadel N, Sun W and Zhou Q. On battery storage system for load pickup in power system restoration[C]. Proc. PES General Meeting Conference \& Exposition, MD, USA, 2014: 1-5.

[11] Teng F, Sun Q, Xie X, et al. A disaster-triggered life-support load restoration framework based on Multi-Agent Consensus System[J]. Neurocomputing, 2015, 170: 339-352.

[12] Rodriguez J R A and Vargas A. Fuzzy-heuristic methodology to estimate the load restoration time in MV networks[J]. IEEE Transactions on Power Systems, 2005, 20(2): 1095-1102.

[13] Gholami A and Aminifar F. A hierarchical response-based approach to the load restoration problem[J]. IEEE Transactions on Smart Grid, 2015, 8(4): 1700-1709.

[14] Kostic T, Germond A J and Alba J J. Optimization and learning of load restoration strategies[J]. International Journal of Electrical Power \& Energy Systems, 1998, 20(2): 131-140.

[15] New York Independent System Operator. Blackout August12, 2003 final report [EB/OL]. .http://www.nyiso.com/ public/ webdocs/media_room/press_releases/2005/ blackout_rpt_final.pdf.

[16] Chen B, Wang $\overline{\mathrm{H}} \mathrm{T}$ and Cao X. Load restoration optimization during the last stage of network reconfiguration considering load fuzzy uncertainty[J]. Automation of Electric Power Systems, 2016, 40(20): 6-12.

[17] Soroudi A and Amraee T. Decision making under uncertainty in energy systems: State of the art[J]. Renewable and Sustainable Energy Reviews, 2013, 28: 376-384.

[18] Soroudi A and Ehsan M. IGDT based robust decision making tool for DNOs in load procurement under severe uncertainty[J]. IEEE Transactions on Smart Grid, 2013, 4(2): 886-895.

[19] Mohammadi-Ivatloo B, Zareipour H, Amjady N, and Ehsan M. Application of information-gap decision theory to risk-constrained self-scheduling of GenCos[J]. IEEE Transactions on Power systems, 2012, 28(2): 1093-1102.

[20] Chen K, Wu W, Zhang B, et al. Robust restoration decision-making model for distribution networks based on information gap decision theory[J]. IEEE Transactions on Smart Grid, 2015, 6(2): 587-597.

[21] Ben-Haim Y. Info-gap decision theory: decisions under severe uncertainty[M]. New York, USA, Academic Press, 2006.

[22] Adibi M M, Borkoski J N, Kafka R J, et al. Frequency response of prime movers during restoration[J]. IEEE Transactions on Power systems, 1999, 14(2): 751-756.

[23] Purchala K, Meeus L, Van Dommelen D, et al. Usefulness of DC power flow for active power flow analysis[C]// IEEE Power Engineering Society General Meeting, 2005: 454-459.

[24] Baran M, Wu F F. Optimal sizing of capacitors placed on a radial distribution system[J]. IEEE Transactions on power Delivery, 1989, 4(1): 735-743.

[25] Farivar M and Low S. H. Branch flow model: Relaxations and convexification-Part I[J]. IEEE Transactions on Power Systems, 2013, 28(3): 2554-2564.

[26] Lipp T, Boyd S. Variations and extension of the convex-concave procedure[J]. Optimization and Engineering, 2016, 17(2): 263287.

[27] Wei W, Wang J, Li N, et al. Optimal Power Flow of Radial Networks and its Variations: A Sequential Convex Optimization Approach[J]. IEEE Transactions on Smart Grid, 2017, 8(6): 2974-2987.

[28] Alizadeh F and Goldfarb D. Second-order cone programming[J]. Mathematical programming, 2003, 95(1): 3-51.

[29] Andersen E D, Roos C and Terlaky T. On implementing a primal-dual interior-point method for conic quadratic optimization[J]. Mathematical Programming, 2003, 95(2): 249-277.

[30] Gu X and Wang D. Investigation on extended black-start schemes of power system considering reasonable load restoration[C]. Proc. PES Power and Energy Engineering Conference (APPEEC), 2014, pp. 1-5. 